Bangladesh J. Bot. 48(2): 367-372, 2019 (June)

\title{
COMPARISON OF MAJOR MEDICINAL COMPONENTS IN THE FIBROUS ROOT OF EPIMEDIUM BREVICORNU MAXIM
}

\author{
JiAn ZaIYOU*, Xu Guifang AND Zhou YaN \\ Henan Institute of Science and Technology, Xinxiang, China, 453003 \\ Collaborative Innovation Center of Modern Biological Breeding, \\ Henan Province-453003, China
}

Keywords: Epimedium brevicornu, Fibrous root, Medical component, RP-HPLC

\begin{abstract}
The contents of main medical components in the fibrous root and medicinal materials of Epimedium brevicornu Maxim was compared. The contents of epimedin $\mathrm{C}$ and icariin in the fibrous root and other parts of E. brevicornu were determined with RP-HPLC. The results showed that there were high contents of medical components in the fibrous root of E. brevicornu. The content of epimedin C in E. brevicornu fibrous root was obviously higher than that of other parts. The content of icariin in E. brevicornu fibrous root was close to that in the rhizome and apparently higher than that in the stem and petiole. The contents of epimedin $\mathrm{C}$ and icariin in the stem (including petiole) were lowest in all parts of E. brevicornu. The fibrous root cut from rhizome when cultivate $E$. brevicornu should be collected and utilized as medicinal materials.
\end{abstract}

\section{Introduction}

Epimedium brevicornu Maxim is a perennial herbaceous plant in Berberidaceae (Flora of China 1979). The leaf of E. brevicornu is usually used as a kind of traditional Chinese medicine in China. There are many types of the medicinal chemical components such as icariin, caohuoside, baohuoside, epimedin A, epimedin B and epimedin C (Li et al. 2005, Meng et al. 2010). The leaf of E. brevicornu is named epimedii folium as traditional Chinese medicine with aphrodisiac, anti-rheumatic and tonic effects. Epimedii folium is usually used to cure impotence, emission, osteomalacia, rheumatism, apoplexy and so on (Chinese Pharmacopoeia Committee 2015).

E. brevicornu is widely distributed in China specially in Taihang Mountain. It is regulated in Chinese Pharmacopoeia that only the leaf of E. brevicornu can be used as Chinese medicine (Chinese Pharmacopoeia Committee 2015). Medicinal materials growers usually reap the aerial part of E. brevicornu when harvest. Then the leaf of E. brevicornu is processed. Now, the resources of E. brevicornu is insufficient after long time harvest. The cultivation technique of E. brevicornu is in study to satisfy the demand of patient on E. brevicornu. Medicinal materials growers usually dug out E. brevicornu plant, then cut off the fibrous root and withered leaf before ramet cultivation according to the immature cultivation technique. Medicinal materials growers think that cutting off the fibrous root of E. brevicornu promote rhizome rooting and germination. It was found that the weight of dry fibrous root is about $1 / 2$ weight of dry aerial part of E. brevicornu after investigation. There is certainly some medical components in the fibrous root of E. brevicornu. The resources of E. brevicornu would be wasted if the fibrous root is given up. Therefore, in the present study the contents of major medical components in the fibrous root and other parts of E. brevicornu were determined and compared. This will provide references for fully usage of E. brevicornu resources in future.

*Author for correspondence: <jian19732004@126.com>. 


\section{Materials and Methods}

Agilent 1260 HPLC instrument, Shimadzu (C18 reverse-phase column, $5 \mu \mathrm{m}, 250 \times 4.6 \mathrm{~mm}$ ), Lectronic Analytic Balance (precision: 0.0001), Ultrasonator and Rotary Evaporator were used in the study.

Ethanol (AR) and acetonitrile (HPLC grade) were used as reagent in experiment. Standard epimedin C and icariin (99.8\%) were purchased from Sichuan Weikeqi Biotechnology Co. Ltd. in China in May 2018.

More than 30 plants of E. brevicornu were randomly dug in Guanshan of Xinxiang city in Henan province China in June 2018. The leaf blade, stem (including petiole), fibrous root and rhizome of these plants were separated and dried to constant weight at $42^{\circ} \mathrm{C}$.

The dry leaf blade, stem (including petiole), fibrous root and rhizome of E. brevicornu were crushed and sieved with 80 meshes sieve. Each material was weighed for $1 \mathrm{~g}$ and extracted with 30 $\mathrm{ml}$ ethanol solvent $(70 \%)$ in the ultrasonic bath for $0.5 \mathrm{hrs}$. The mixture was filtered with filter paper. The residue was extracted with the same solvent $(30 \mathrm{ml}$ of $70 \%$ ethanol) and filtered once again. These filtrates were mixed and added to $60 \mathrm{ml}$. The extract was filtered with $0.22 \mu \mathrm{m}$ membrane filter. Each kind of materials was extracted three times.

Standard icariin solutions were prepared at $0.00065,0.0031,0.0062,0.0315,0.31$ and 0.5 $\mathrm{mg} / \mathrm{ml}$, respectively. Standard epimedin C solutions were prepared at $0.001,0.005,0.01,0.05$ and $0.5 \mathrm{mg} / \mathrm{ml}$, respectively.

The Diamonsil C18 reverse-phase column $(5 \mu \mathrm{m}, 250 \times 4.6 \mathrm{~mm})$ was used as HPLC column. The volume of extract injected was $10 \mu$. The gradient mobile phase consists of acetonitrile and water. The content ( $\mathrm{v} / \mathrm{v})$ of acetonitrile in the gradient mobile phase varied from 22 to $29 \%$ in 0 - 12 min, 29 to $29.5 \%$ in $12-20 \mathrm{~min}$ and 29.5 to $30 \%$ in $20-22 \mathrm{~min}$. The flow rate of mobile phase was $1 \mathrm{ml} / \mathrm{min}$ and the temperature in HPLC column was $35^{\circ} \mathrm{C}$. A variable wavelength recorder was set at $270 \mathrm{~nm}$ to detect ingredients eluted from the column.

These standard solutions and those prepared extracts were respectively analyzed according to the above HPLC method. Chromatography peak areas of epimedin C and icariin in each chromatogram were respectively, recorded.

All those prepared extracts were analyzed according to the above HPLC method. Chromatography peak areas of icariin and epimedin $\mathrm{C}$ in extracts were recorded, respectively. These contents of icariin and epimedin $\mathrm{C}$ in extracts were analyzed according to their chromatography peak areas and the standard curves (relating these peak areas to their contents). All data were analyzed with SPSS (Statistical Product and Service Solutions).

\section{Results and Discussion}

The HPLC chromatograms of standard epimedin $C$ and icariin are shown in Fig. 1. These standard curves of icariin and epimedin $\mathrm{C}$ were drafted according to their peak areas and their contents (Table 1 and Fig. 2).

The peaks of epimedin $\mathrm{C}$ and icariin in extract chromatograms were identified according to their retention time in HPLC (Fig. 3). The concentrations of epimedin C and icariin in extracts were analyzed according to their peak areas and standard curves also (Table 2). Then the contents of epimedin $\mathrm{C}$ and icariin in E. brevicornu materials were analyzed according the methods of preparation extract.

It can be seen from the results that there were high contents of medical components in the fibrous root of E. brevicornu. The content of epimedin $\mathrm{C}$ in the fibrous root was higher than that in other parts including leaf blade. The content of icariin in the fibrous root is close to that in the 
rhizome and higher than that in the stem and petiole, although lower than that in the leaf blade (Tables 2,3). The contents of epimedin C and icariin in the stem (include petiole) are lowest in all parts of E. brevicornu.
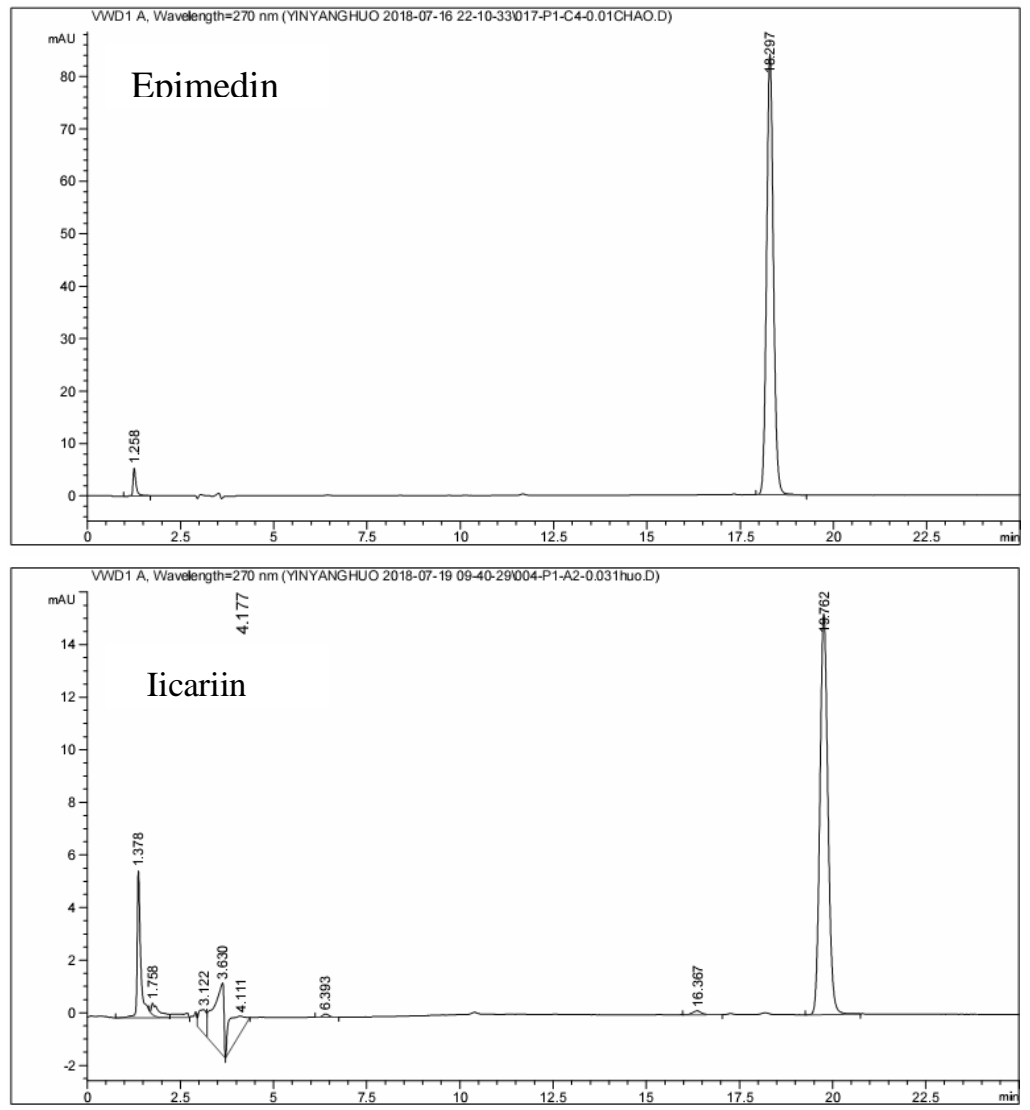

Fig. 1. HPLC chromatograms of standard epimedin C and icariin.

Table 1. Analysis results of standard icariin and epimedin C.

\begin{tabular}{cccc}
\hline \multicolumn{2}{c}{ Epimedin C (Retention time 18.297 min) } & \multicolumn{2}{c}{ Icariin (Retention time $19.762 \mathrm{~min})$} \\
\hline $\begin{array}{c}\text { Concentration } \\
(\mathrm{mg} / \mathrm{ml})\end{array}$ & Peak area & $\begin{array}{c}\text { Concentration } \\
(\mathrm{mg} / \mathrm{ml})\end{array}$ & Peak area \\
\hline 0.001 & 100.08 & 0.00062 & 4.5858 \\
0.005 & 498.53 & 0.0031 & 21.877 \\
0.01 & 1063.24 & 0.0062 & 45.351 \\
0.05 & 5276.14 & 0.031 & 227.97 \\
0.5 & 63782.61 & 0.31 & 2437.29 \\
& & 0.5 & 3930.85 \\
\hline
\end{tabular}




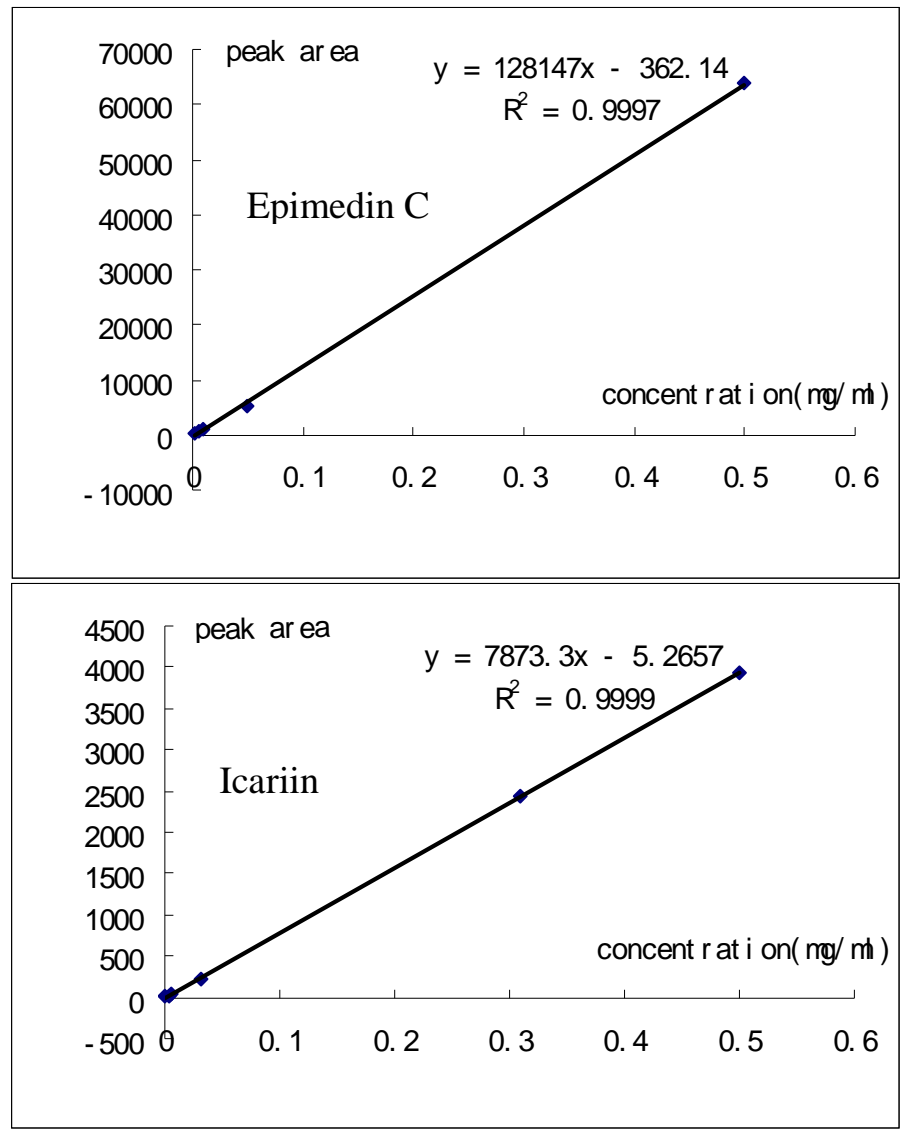

Fig. 2. Standard curves of icariin and epimedin C.

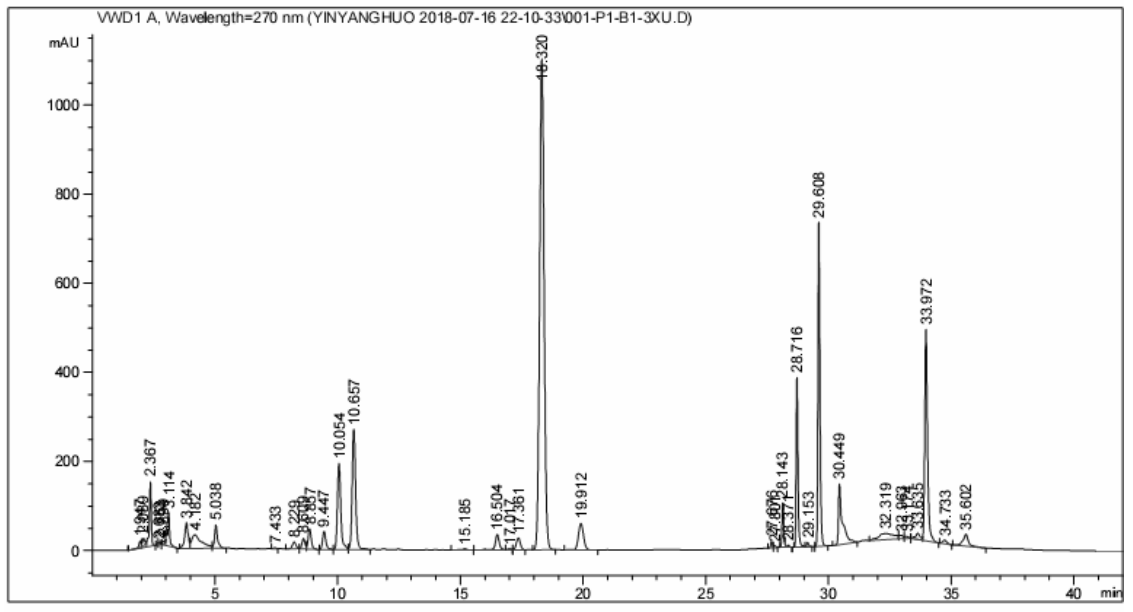

Fig. 3. HPLC chromatograms of E. brevicornu materials. 
Table 2. Contents of epimedin $\mathrm{C}$ and icariin in E. brevicornu materials.

\begin{tabular}{|c|c|c|c|c|}
\hline \multirow[t]{2}{*}{ Parts } & \multicolumn{2}{|c|}{ Epimedin $\mathrm{C}$} & \multicolumn{2}{|c|}{ Icariin } \\
\hline & $\begin{array}{l}\text { Peak } \\
\text { area }\end{array}$ & $\begin{array}{l}\text { Content } \\
(\mathrm{mg} / \mathrm{g})\end{array}$ & $\begin{array}{l}\text { Peak } \\
\text { area }\end{array}$ & Content \\
\hline \multirow[t]{3}{*}{ Fibrous root } & 13064.62 & 6.287 & 1079.91 & 8.270 \\
\hline & 13746.01 & 6.606 & 1064.21 & 8.150 \\
\hline & 13931.81 & 6.693 & 1083.59 & 8.298 \\
\hline \multirow[t]{3}{*}{ Rhizome } & 1461.93 & 0.855 & 925.69 & 7.135 \\
\hline & 1507.81 & 0.876 & 954.24 & 7.352 \\
\hline & 1504.70 & 0.875 & 945.53 & 7.286 \\
\hline \multirow[t]{3}{*}{ Stem and petiole } & 376.99 & 0.347 & 140.33 & 1.110 \\
\hline & 379.09 & 0.348 & 143.74 & 1.136 \\
\hline & 392.72 & 0.354 & 134.04 & 1.062 \\
\hline \multirow[t]{3}{*}{ Leaf blade } & 2625.49 & 1.399 & 3051.12 & 23.292 \\
\hline & 2537.07 & 1.358 & 2763.01 & 21.096 \\
\hline & 2499.78 & 1.341 & 2899.86 & 22.139 \\
\hline
\end{tabular}

Table 3. Multiple comparisons of medical components contents in different parts.

\begin{tabular}{lclc}
\hline \multicolumn{2}{c}{ Epimedin C } & \multicolumn{2}{c}{ Icariin } \\
\hline Parts & $\begin{array}{c}\text { Mean contents } \\
\text { comparison* }(\mathrm{mg} / \mathrm{g})\end{array}$ & Parts & $\begin{array}{c}\text { Mean contents comparison* } \\
(\mathrm{mg} / \mathrm{g})\end{array}$ \\
\hline Fibrous root & $6.5288^{\mathrm{a}}$ & Leaf blade & $22.1757^{\mathrm{a}}$ \\
Leaf blade & $1.3660^{\mathrm{b}}$ & Fibrous root & $8.2393^{\mathrm{b}}$ \\
Rhizome & $0.8685^{\mathrm{c}}$ & Rhizome & $7.2577^{\mathrm{b}}$ \\
Stem and petiole & $0.3495^{\mathrm{d}}$ & Stem and petiole & $1.1027^{\mathrm{c}}$ \\
\hline
\end{tabular}

The mean difference is significant at the 0.01 level.*The different letters indicate there is obvious difference between these means, The same letters indicates there is not obvious difference between these means.

There are high contents of medical components in the fibrous root of E. brevicornu. The contents of some medical components such as epimedin $\mathrm{C}$ are higher than that in medicinal materials from E. brevicornu. The fibrous root of E. brevicornu is usually cut off the rhizome before ramet cultivation to promote rhizome tooting and germination. The fibrous root is often given up by medicinal materials growers. Thus, the resources of E. brevicornu is seriously wasted. It may be suggested that the fibrous root cut from rhizome when cultivate E. brevicornu should be collected and utilized as medicinal materials. The fibrous root can also be used to extract medical components to reduce the waste of E. brevicornu resources.

It is regulated in Chinese Pharmacopoeia that the part of E. brevicornu used as Chinese medicine is leaf (Chinese Pharmacopoeia Committee 2015). But it is not clear that the medicinal materials come from E. brevicornu includes blade or not. It was found that the contents of medical components in the stem and petiole of E. brevicornu were very low according to the result of the present study. This result is consistent with that of Sun Chao on the whole (Sun et a.l 2006, Chu 
et a.l 2007). The weight of dry stem (including petiole) is about $1 / 2$ weight of dry leaf of E. brevicornu. The stem and petiole of E. brevicornu mixed in the medicinal materials reduce its quality. Therefore, epimedii folium should not include stem and petiole.

\section{Acknowledgements}

This study was supported by Science and Technology research project of Henan province (NO. 182102310634, NO. 152102210309).

\section{References}

Chinese Pharmacopoeia Committee 2015. Pharmacopoeia of the People's Republic of China, 2015ed, PartI. Chemical Industry Press, Beijing, 68-69.

Chu Rong, LIN Chang-hu, SUN Chao, ZHU Li and ZHONG Yan 2007. Study on the change of Glucoside Content of Two Species of Epimedium Imaginary Root in Seasons. Seed 26(1): 30-31.

Flora of China Committee 1979. Flora of China (volume 27). Science Press, Beijing, 296-299.

Li Yubo, MENG Fanhao, LU Xiumei and LI Famei 2005. Chemical constituents from herb of Epimedium brevicornum. China J Chinese Materia Medica 30(8): 586-588.

Meng Ning, KONG Kai and LI Shiweng 2010. Advancesin Studieson Chemical Constituents and Pharmaceutical Activitiesin Species of Epimedium. Acta Bot. Borea1 Occident. Sin. 30(5): 1063-1073.

Sun Chao, ZHANG Yong-min, LIN Chang-hu, ZOU Jian-ling and ZHONG Yan 2006. Study on the change ofG lycoside Content ofEpmi ediumAcum inatim. Chin. J MAP 23(8): 769-771.

(Manuscript received on 11 September, 2018; revised on 19 December, 2018) 